\title{
Value-Based Purchasing for Hospital-Acquired Venous Thromboembolism: Too Much, Too Soon
}

\author{
Christopher G. Roy, MD, MPH*
}

Mount Auburn Hospital, Cambridge, Massachusetts; Harvard Medical School, Cambridge, Massachusetts, Massachusetts Institute of Technology, Cambridge, Massachusetts.

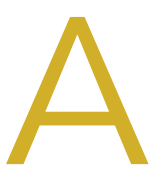

s a hospital-acquired condition responsible for a significant share of preventable deaths in the United States, ${ }^{1}$ venous thromboembolism (VTE) prevention should remain a high priority for healthcare organizations. Pursuant to the goal of reducing the frequency of this and other hospital-acquired conditions, several performance measures have been developed by third-party payers in the United States to provide incentives for inpatients to receive prophylaxis measures appropriate to their specific level of risk. Perhaps the best known of these is the Hospital Value-Based Purchasing Program, initiated by the Center for Medicare and Medicaid Studies (CMS) in 2013 as a provision of the Affordable Care Act. ${ }^{2}$ The Joint Commission, as steward of the 6 VTE-related measures, ${ }^{3}$ dictates the criteria for assessing performance. However, recent adjustments to one of these measures have been performed in such a way that neglects real-world considerations faced by providers and threatens to delegitimize the important role that value-based purchasing should have in reimbursement.

Effective in 2017, the guidelines pertaining to abstraction-based reporting added a new component to the VTE-6 measure, which applies to those inpatients not ordered to receive mechanical or pharmacologic prophylaxis who go on to suffer VTE. Specifically, it is concerned with how accurately hospitals stratify such patients as low risk before the decision is made to not order either method of prophylaxis. With the update, to satisfy the measure, a formal assessment confirming a patient's low-risk status must have been documented between arrival and the time the VTE diagnostic test was performed. The guidelines explicitly note that only 3 risk assessment models (RAMs) are accepted, including the Caprini DVT Prediction Score, Padua Prediction Score, and IMPROVE VTE Risk Score. ${ }^{4}$ The rationale for this addition to the measure clearly is to protect patients from being incorrectly designated as low risk and subsequently receiving inadequate prophylaxis that could increase their likelihood of developing preventable VTE. Unfortunately, in its current form, it imposes a substantial burden on providers and healthcare organiza-

*Address for correspondence: Christopher G. Roy, MD, MPH; Hospitalist Office, 330 Mount Auburn Street, Cambridge, MA 02138; Telephone: 617-4995112; Fax: 617-575-8608; Email: cgroy@mah.harvard.edu

Published online first April 25, 2018.

Received: December 22, 2017; Revised: February 2, 2018; Accepted: February 9, 2018

๔ 2018 Society of Hospital Medicine DOI 10.12788/jhm.2969 tions, without much promise of significantly reducing rates of this pervasive threat to patient safety.

\section{LIMITATIONS}

Although the aim of reducing the incidence of VTE is laudable, this updated requirement for VTE-6 is problematic on several levels. First, there is considerable uncertainty regarding how to implement the RAMs clinically in a user-friendly way that is conducive to their intended use. Due to limitations in most computerized physician order entry systems, it is not feasible to mandate the RAMs for only those patients not ordered for VTE prophylaxis (nor would it be sensible to restrict performing the assessment to low-risk patients, as the point of RAMs is to help risk stratify and not simply validate whatever determinations were already made by other means). As virtually every class of inpatient has some risk of VTE development, these factors effectively require that a score be tabulated on all admitted patients, giving the measure an enormous footprint on clinical operations. This is important because the permissible RAMs can sometimes be quite burdensome to complete faithfully. For instance, the Caprini Score necessitates the fairly prodigious collection and input of up to 26 data points. Some of the questions require exceedingly granular data, such as whether there is any "history of unexplained stillborn infant, recurrent spontaneous abortion (more than 3), premature birth with toxemia or growth restricted infant." ${ }^{\prime}$ This clearly is far outside the scope of most focused admission assessments. Already deluged with the number of clicks inherent to the workflow of most electronic health records, ${ }^{6}$ it seems likely that some providers default to selecting "no" for such prompts as a time-saving measure, potentially sabotaging the goal of linking patients with a risk-appropriate method of prophylaxis. Meanwhile, those who are diligent about completing the assessment honestly will find themselves rewarded with less time to dedicate to other critical aspects of patient care. ${ }^{7}$

The small number of RAMs accepted under the measure also fails to account for the breadth of clinical circumstances providers faced. Although the permitted models are validated in certain patient populations, they exclude some that might be better suited for many practice environments. The University of California San Diego "3 bucket" design, for instance, has been shown to result in high levels of risk-appropriate prophylaxis, has high inter-user agreement, and perhaps most importantly, is relatively quick and easy to use. ${ }^{8}$ Also critical, it is easier to integrate into the admission workflow for under-resourced hospitals that might not have the ability to incorporate a point-based 
risk score calculator into their electronic health records.

Finally, the relative abruptness with which the changes were made complicated the task for institutions to integrate the RAMs into their applicable order sets in a user-friendly fashion. The new guidelines were released only 6 months before taking effect, ${ }^{9}$ and the RAM requirement was not widely advertised. This left a fairly short window that does not seem to reflect an understanding by the Joint Commission of the process required by hospitals to make such a transition responsibly. This should involve obtaining inputs from multiple specialty stakeholders on which RAM to employ, working with information system specialists on how to restructure key order sets, and education of end-users on how to apply them correctly. ${ }^{10}$

\section{RECOMMENDATIONS}

For these reasons, the rollout of the VTE-6 update falls well short of its ambitions. Satisfying the measure necessitates a substantial investment of time and effort by providers and yet forcing the use of such decidedly imperfect RAMs could paradoxically worsen accurate risk stratification and appropriate use of prophylaxis. Also, while it represents only a small slice of pay-for-performance initiatives, its broader impact should not be underestimated. Unlike many of the more specific items, the VTE measures affect the workflow related to virtually all hospitalized patients. Therefore, it is imperative that regulators "get it right," as it might only take one poorly conceived mandate of this type to risk permanently souring providers and hospitals on the idea of value-based purchasing. The Joint Commission and CMS ought to seriously consider retracting the new provisions until the role of RAMs for VTE prevention is better understood. This would buy time to reconfigure the measure in a way that is compatible with actual clinical care and for hospitals to thoughtfully design how new requirements can best be implemented.

Disclosurses: The author has nothing to disclose.

\section{References}

1. Clagett GP, Anderson FA Jr, Heit J, Levine MN, Wheeler HB. Prevention of venous thromboembolism. Chest. 1995;108(4 Suppl):312S-334S.

2. Center for Medicare and Medicaid Studies. Hospital value based purchasing. https://www.cms.gov/Outreach-and-Education/Medicare-Learning-Network-MLN/MLNProducts/downloads/Hospital_VBPurchasing_Fact_ Sheet_ICN907664.pdf. Accessed December 18, 2017.

3. The Joint Commission. Specifications manual for national hospital inpatient quality measures. https://www.jointcommission.org/specifications_manual_ for_national_hospital_inpatient_quality_measures.aspx. Accessed December 18, 2017.

4. The Joint Commission. Specifications manual for national hospital inpatient quality measures. https://www.jointcommission.org/specifications_manual_ for_national_hospital_inpatient_quality_measures.aspx. Accessed December 18, 2017.

5. Venous Resource Center. Caprini score: DVT risk assessment. https://venousdisease.com/dvt-risk-assessment-online. Accessed December 19, 2017.

6. Hill RG, Sears LM, Melanson SW. 4000 Clicks: A productivity analysis of electronic medical records in a community hospital ED. Am J Emerg Med. 2013;31(11):1591-1594.

7. Clynch N, Kellett J. Medical documentation: Part of the solution, or part of the problem? A narrative review of the literature on the time spent on and value of medical documentation. Int J Med Inform. 2015;84(4):221-228.

8. Maynard GA, Morris TA, Jenkins $I H$, et al. Optimizing prevention of hospital-acquired venous thromboembolism (VTE): Prospective validation of a VTE risk assessment model. J Hosp Med. 2010;5(1):10-18.

9. The Joint Commission. Specifications manual for national hospital inpatient quality measures release notes v5.2. Available at: https://www.jointcommission.org/specifications_manual_for_national_hospital_inpatient_quality_measures.aspx. Accessed December 18, 2017.

10. Agency for Healthcare Quality and Research. Preventing hospital acquired venous thromboembolism: A guide for effective quality improvement. Available at: https://www.ahrq.gov/sites/default/files/publications/files/vteguide pdf. Accessed December 18, 2017. 